\title{
Analysis of noise spectra in GaAs and GaN Schottky barrier diodes
}

\author{
D Pardo ${ }^{1}$, J Grajal ${ }^{1}$, B Mencía ${ }^{1}$, S Pérez ${ }^{2}$, J Mateos ${ }^{2}$ and T \\ González ${ }^{2}$ \\ ${ }^{1}$ Department of Senales, Sistemas y Radiocomunicaciones. Universidad Politécnica \\ de Madrid, Av. Complutense s/n. 28040 Madrid, Spain \\ ${ }^{2}$ Department of Física Aplicada. Universidad de Salamanca, Plaza de la Merced s/n. \\ 37008 Salamanca, Spain \\ E-mail: dpardo@gmr.ssr.upm.es
}

\begin{abstract}
The Monte Carlo method is applied in this paper to characterize the noise spectra of GaAs and GaN Schottky barrier diodes operating under static and time varying conditions. We show the influence of the structure of the diode and working regimes on the noise spectrum of the diodes. Besides, the paper evaluates the capabilities of published analytical models to describe the noise spectra in Schottky diodes under time varying conditions. This is a further step towards the development of a design tool that integrates both the electrical response and the intrinsic noise generated in the devices.
\end{abstract}




\section{Introduction}

The terahertz region of the electromagnetic spectrum presents a wide range of applications such as molecular spectroscopy, radio-astronomy, earth observation, security, radar, etc $[1,2,3]$. However, the development of terahertz technologies is being conditioned by the small number of compact, powerful and tunable $\mathrm{THz}$ sources and detectors working at room temperature $[4,5]$. Schottky barrier diodes (SBD) are the preferred devices operating as sensors and sources in the $\mathrm{THz}$ region at room temperature, due to its inherent simplicity, the reasonable sensitivity and low noise of these devices operating as detectors [6] and the high efficiency of $\mathrm{THz}$ multiplier chains based on SBDs $[4,7]$.

GaAs is the most widely used semiconductor in SBDs because of its high electron mobility, necessary for $\mathrm{THz}$ applications, and the maturity of the associated technology [4]. Multi-anode frequency multipliers have been introduced to increase the power handling capabilities at millimeter and submillimeter wavelengths. However, the available power at the final stage of the frequency multiplier chains is limited by the breakdown voltage and the heating of the GaAs diodes in the first stages of the chains $[5,7]$. To achieve higher power levels at sub- THz frequencies, the fabrication of GaN based SBDs increases the breakdown voltage and subsequently greatly increases the power handling capabilities of frequency multipliers. However, results from numerical simulations of a $200 \mathrm{GHz}$ doubler based on SBDs have shown the lower conversion efficiency of GaN compared to GaAs based Schottky multiplier, due to the lower electron mobility of GaN than GaAs [8].

An accurate description of the electrical response of these devices must evaluate the relevance of the fluctuations in the voltages and currents at the terminals of the devices. These fluctuations, known as electrical noise, suppose a limit in the detection and generation of signals in sensors and sources, respectively [9]. The characterization of these fluctuations and the study of the noise sources are fundamental aspects in the design of electrical devices, especially in the $\mathrm{THz}$ region, where the high frequency physical effects can degrade the noise characteristics of the devices. The joint analysis of both the electrical response and the intrinsic noise is required to obtain accurate designs of devices.

The Monte Carlo method applied to the description of the charge transport in semiconductor devices has demonstrated to be the most rigorous technique for the analysis of the electrical response and the intrinsic noise of the devices, since it naturally incorporates the microscopic noise sources present in the devices. A lot of efforts have been dedicated to understand and analyze the noise features of SBDs employing this technique, especially GaAs based devices [10, 11, 12]. However only a partial analysis of the noise spectra of GaAs based SBDs has been performed. On the other hand, the study of the noise spectra of GaN based SBDs is an unexplored topic that is carried out in this paper.

This paper presents a complete analysis of the noise spectra of GaAs and GaN 
based SBDs operating under static and time varying conditions. A systematic study of the dependence of the intrinsic noise with the applied voltage and the device structure is carried out employing the classic Monte Carlo method and published analytical models. The analysis of the noise spectra based on MC simulations performed along the paper are also used to determine the accuracy and the limit of validity of the analytical model published in [13] for the high-frequency noise in SBDs.

The paper begins with a brief summary of the main characteristics of the MC simulator employed in this work and published analytical models for the noise spectra in SBDs. The characteristics of the SBD taken as a reference for this study are presented in section 3. The main features of noise spectra generated in GaAs and GaN based SBDs under static conditions are analyzed in section 4, evaluating their dependence on the device structure and bias. Both MC simulations and the predictions from the analytical models are presented. Section 5 is devoted to studying the performance of the noise spectra of GaAs and GaN based SBDs under time varying conditions. It analyzes the possibilities to apply analytical models to describe these noise spectra. The study performed in sections 4 and 5 is carried out at $300 \mathrm{~K}$, and section 6 is dedicated to study the impact of self-heating on noise spectra. Some conclusions are drawn in section 7 .

\section{Monte Carlo simulator and analytical models}

Noise analysis have been carried out by using an ensemble MC simulator three dimensional in momentum space, which is self-consistently coupled with a one dimensional Poisson solver. The effect of degeneracy is accounted for by locally using the classic rejection technique $[14,15]$. The ohmic contact is modeled as a surface that injects carriers in thermal equilibrium with the lattice (in order to maintain the neutrality in the region very close to the contact), according to Fermi- Dirac statistics. In addition, any carrier reaching the contact leaves the device. On the other hand, the Schottky contact is simulated as a perfect absorbent surface. Scattering mechanisms included in the Monte Carlo simulation are ionized impurities, acoustic phonon, polar and non-polar optic phonon and intervalley mechanisms, for both GaAs and GaN semiconductors. The band structure is modeled as a conduction band with three spherical non-parabolic valleys [14]. The charge density is updated every $0.5 \mathrm{fs}$ and devices are divided into equal cells of $20 \AA$ large. The number of simulated carriers is around $10^{5}$. The time evolution of the current fluctuations provided by Monte Carlo simulations are analyzed employing the time correlation function. The spectral density of the current fluctuations is obtained from the Fourier transform of the correlation function [11].

Reference [13] provides an analytical model for the high frequency noise in SBDs under constant voltage conditions. In this model, the fluctuations of the current at the terminals of the SBD are interpreted as the result of the dynamics of uncompensated charge formed in the borders of the regions characterized by abrupt changes of the free carrier concentration. According to this model, the abrupt change of the free carrier concentration in the $n^{+}-n$ homojunction is the responsible of the generation 


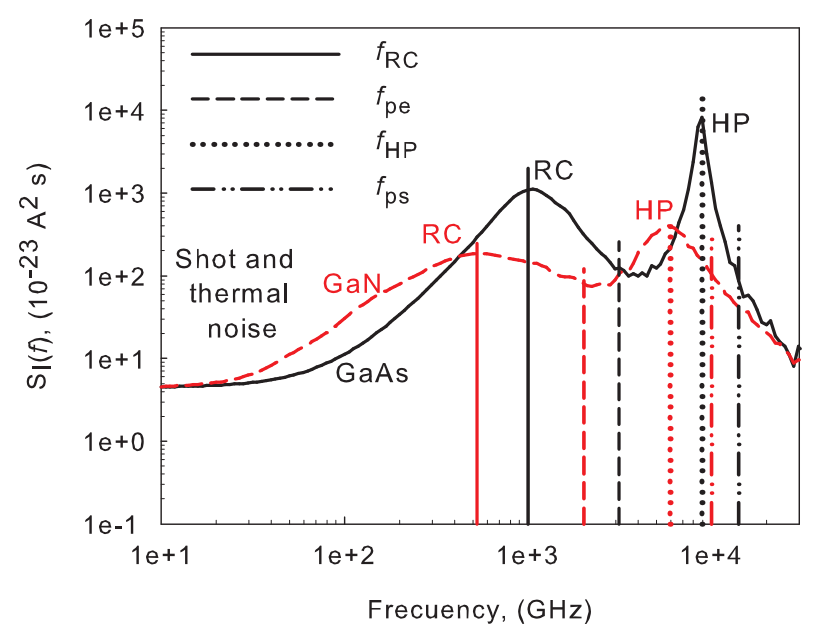

Figure 1. Identification of the main features of the noise spectra of GaAs and GaN SBDs defined in section 3, under applied voltages $V_{D C}=0.80 \mathrm{~V}$ and $V_{D C}=0.97 \mathrm{~V}$ respectively. The vertical lines in the spectra identify the central frequency of the RC resonance $\left(f_{R C}\right)$, the plasma frequency of the epilayer $\left(f_{p e}\right)$, the central frequency of the HP resonance $\left(f_{H P}\right)$ and the plasma frequency of the substrate $\left(f_{p s}\right)$.

of a high frequency peak, usually known as hybrid plasma peak, (notation: HP resonance and $f_{H P}$ for the central frequency of this resonance), since it is centered at the intermediate frequency of the plasma frequency of the substrate and the epilayer, given by $f_{p s}=\left[q^{2} N_{s} /\left(\epsilon_{0} \epsilon_{r} m^{*}\right)\right]^{1 / 2} /(2 \pi)$ and $f_{p e}=\left[q^{2} N_{e} /\left(\epsilon_{0} \epsilon_{r} m^{*}\right)\right]^{1 / 2} /(2 \pi)$, respectively. In these expressions q represents the absolute value of the electron charge, $\epsilon_{0} \epsilon_{r}$ the permittivity of the semiconductor, $N_{s}$ and $N_{e}$ are the the doping concentration of the substrate and the epilayer, respectively. The abrupt change of the free carrier concentration in the depletion region gives rise to a resonance in the intermediatehigh frequency region of the noise spectrum, known as the returning carrier resonance, (notation: $\mathrm{RC}$ resonance and $f_{R C}$ for the central frequency of this resonance). The name of this peak becomes from a previous interpretation published in 1986 [16], where the $\mathrm{RC}$ resonance is interpreted as fluctuations in the current originated by those electrons in the depletion region that approach the Schottky barrier with insufficient energy to surmounting it and they return back to the neutral region of the epilayer. Figure 1 shows the noise spectra for the SBD described in next section, based on GaAs and GaN semiconductor operating under static conditions, $\left(V_{D C}=0.80 \mathrm{~V}\right.$ for GaAs SBD and $V_{D C}=0.97 \mathrm{~V}$ for GaN SBD to obtain similar values of current flowing in those SBDs). The RC and the HP resonances are identified in this figure. The plasma frequency of the substrate and the epilayer of the GaAs and GaN reference SBD are also shown, (the doping concentrations and lengths for the substrate and the epilayer of the reference SBD can be observed in figure 2).

Employing the Langevin equation to describe the dynamics of the fluctuations of the free carriers in the $n^{+}$and $n$ regions and the Nyquist theorem, reference [13] develops an analytical expression for the spectral density of the current fluctuations, 
$S_{I}(f)$. This approach considers the self-consistent actualization of the electric field with the fluctuations of the charge. The general expression developed for $S_{I}(f)$ can be simplified assuming a constant mobility in the $n^{+}$and $n$ regions

$$
S_{I}(f)=4 k_{B} T 2 \pi f_{s c} C f^{2} \frac{r_{s} f_{p s}^{2} \phi\left(f_{p e}\right)+r_{e} f_{p e}^{2} \phi\left(f_{p s}\right)}{\phi\left(f_{H P}\right) \phi\left(f_{R C}\right)}
$$

where the notation of the regions of the SBD is shown in figure 2, $C=\epsilon_{0} \epsilon_{r} A / L$ is the geometrical capacitance of the SBD, A is the cross section area of the diode, $f_{s c}=q /\left(2 \pi m^{*} \mu\right)$ is the carrier momentum relaxation rate, $m^{*}$ the effective mass of the electrons and $\mu$ the low field electron mobility; $r_{i}=l_{i} / L(i=s, e, d)$ is the relative length of the i-region, $\phi\left(f_{i}\right)=\left(f^{2}-f_{i}^{2}\right)^{2}+f_{s c}^{2} f^{2} ; f_{p s}$ and $f_{p e}$ are the plasma frequency of the substrate and epilayer respectively. When $l_{d}<L-l_{s}, f_{H P}$ and $f_{R C}$, the frequencies of the hybrid plasma and returning carriers resonances respectively, can be approximated by

$$
\begin{aligned}
& f_{H P}^{2}=f_{h}^{2}-\beta f_{p e}^{2} r_{d} \\
& f_{R C}^{2}=(1+\beta) f_{p e}^{2} r_{d}
\end{aligned}
$$

where $f_{h}^{2}=f_{p s}^{2}\left(r_{e}+r_{d}\right)+f_{p e}^{2} r_{s}$ and $\beta=r_{s}\left(f_{p s}^{2}-f_{p e}^{2}\right) / f_{h}^{2}=r_{s}\left(N_{s}-N_{e}\right) /\left[N_{s}\left(1-r_{s}\right)+N_{e} r_{s}\right]$.

The accuracy of this model is limited by the assumption that changes of the free carrier concentration in the $n^{+}-n$ interface and near the edge of the depletion region are abrupt; it also assumes that the depletion region is empty, so shot noise is not considered and, therefore, the model fails in the low frequency region of noise spectrum. Besides, it neglects the potential drop in the $n^{+}-n$ interface.

Together with MC simulations, the general expression developed in [13] for $S_{I}(f)$ as well as previous equations (1) to (3) describe the high-frequency region of the noise spectra of SBDs, (RC and HP resonances). The model published in [16] for shot and thermal noise is employed to interpret the low frequency region of the noise spectra obtained from MC simulation of SBDs.

\section{Devices structure}

As a reference, the selected Schottky diode has an epilayer length of $350 \mathrm{~nm}$ with a doping concentration of $10^{17} \mathrm{~cm}^{-3}$. The length of the substrate is $500 \mathrm{~nm}$, which has been shortened as compared to the typical values of fabricated devices in order to reduce the computational cost of the Monte Carlo method. The doping concentration of the substrate is $210^{18} \mathrm{~cm}^{-3}$. The anode area is $36 \mu \mathrm{m}^{2}$. The ideal barrier height has been set at $0.99 \mathrm{~V}$ for GaAs and $1.20 \mathrm{~V}$ for GaN diodes, respectively. The technological parameters of the reference SBD has been selected according to fabricated $\mathrm{SBD}$ multipliers operating in the near $\mathrm{THz}$ region where the application of GaN SBDs is justified [17].

Figure 3 shows the I-V and C-V curves obtained from Monte Carlo simulations of GaAs and GaN reference SBDs at room temperatures between $300 \mathrm{~K}$ and $600 \mathrm{~K}$. For 


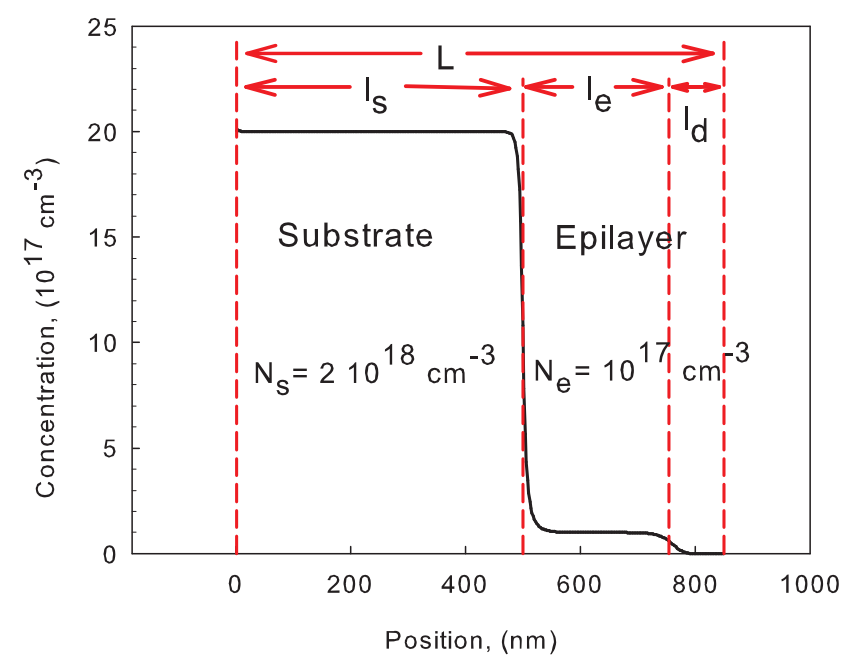

Figure 2. Schematic representation of the $n^{+}-n$ reference SBD, showing the notation employed for the different regions of the SBD.

voltages lower than the built-in potential $\left(V_{b i}(T), 0.95 \mathrm{~V}\right.$ for GaAs and $1.11 \mathrm{~V}$ for GaN at $300 \mathrm{~K}$ ) the $\mathrm{I}-\mathrm{V}$ curves show the typical non linear conductance region associated to thermionic emission through Schottky barrier. For $V>V_{b i}$, the current curves show a linear dependence with the applied voltage, which is associated to an ohmic behavior. The capacitance-voltage curves have been obtained as variations in the average number of carriers inside the diode with the applied voltage.

\section{Noise under static conditions}

In this section we present an analysis of the main features of the noise spectra of GaAs and GaN SBDs under static conditions, employing the MC method and the analytical models of [13] and [16]. The low frequency region of the noise spectra, limited by the enhancement of the $\mathrm{RC}$ resonance, is studied in subsection 4.1. The $\mathrm{RC}$ and the $\mathrm{HP}$ resonances are analyzed in subsections 4.2 and 4.3, respectively. To analyze the influence of the HP resonance in the performance of the $\mathrm{RC}$ resonance, we have simulated homogeneous SBDs, that is, SBDs without substrate. Since for homogeneous SBDs the $n^{+}-n$ homojunction does not exist, the HP resonance, which is related to the $n^{+}-n$ homojunction, is not present in the noise spectra.

\subsection{Low frequency noise}

Figure 4 shows the noise spectral density of the reference SBDs based on GaAs (black symbols) and GaN (red symbols) as a function of the current flowing in the SBDs at $f=0, S_{I}(0)$. The Monte Carlo simulator employed to obtain these data neither implement generation-recombination phenomena nor $1 / \mathrm{f}$ noise. Therefore, $S_{I}(0)$ 

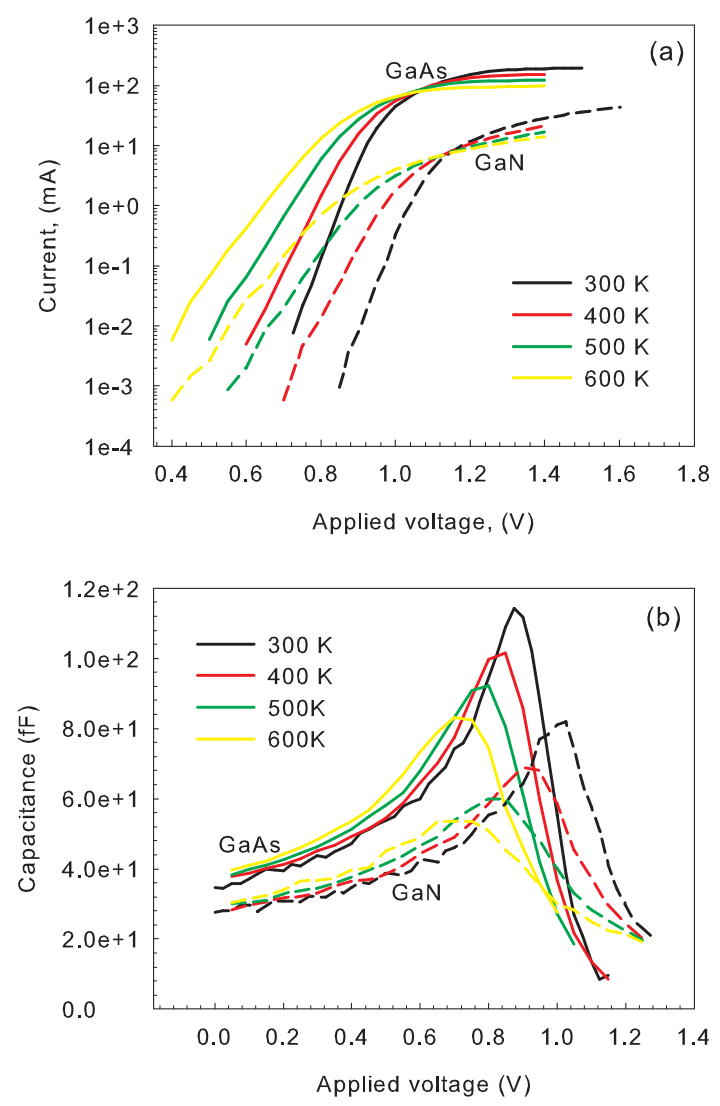

Figure 3. I-V curves (a) and C-V curves (b) for GaAs and GaN reference SBDs at different room temperatures obtained from MC simulations

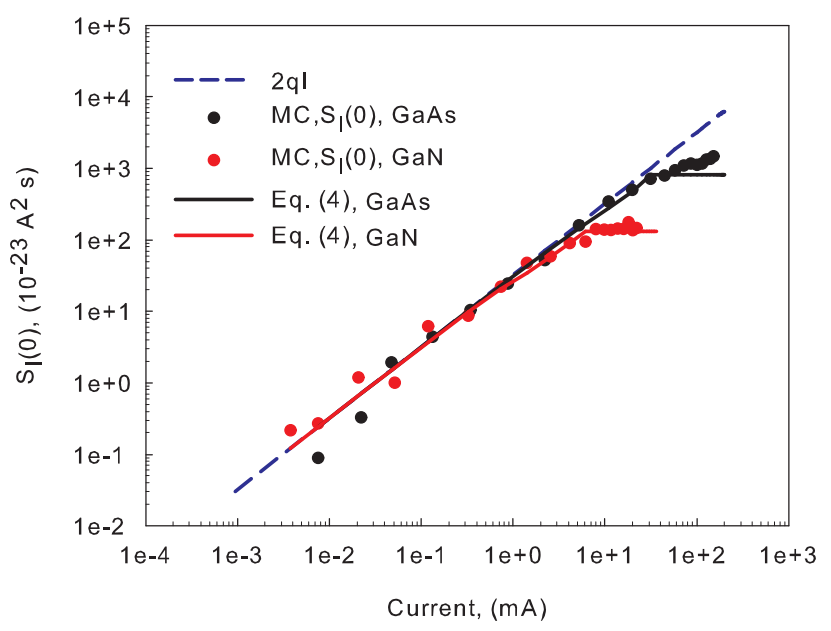

Figure 4. Low frequency noise for GaAs and GaN $n^{+}-n$ reference SBDs as a function of the current flow through the diodes. Symbols represent the results from Monte Carlo simulations and continuous lines correspond to (4) 
provided by the simulator is in accordance with the analytical expression given in [16]

$$
S_{I}(0)=\frac{2 q I R_{j}^{2}}{\left(R_{s}+R_{j}\right)^{2}}+\frac{4 K_{B} T R_{s}}{\left(R_{s}+R_{j}\right)^{2}}
$$

where $R_{s}$ is the series resistance of the substrate and non-depleted region of the epitaxial layer and $R_{j}$ represents the junction resistance. $R_{s}$ is evaluated from the slope of the linear region of the I-V characteristic of figure 3 for applied voltages $V>V_{b i}$. It takes values about $1.8 \Omega$ and $12.1 \Omega$ for GaAs and GaN reference SBDs respectively.

From this expression, for $V<V_{b i}$ corresponding to $R_{j}>>R_{s}$, the low frequency spectral density, $S_{I}(0)$, behaves as $2 \mathrm{qI}$, the shot-noise law. This noise is due to electrons crossing randomly the Schottky barrier [16]. The spectral density of the current fluctuations originated by these electrons is white up to frequencies on the order of the reciprocal transit time and drops steeply at higher frequencies. For $V>V_{b i}$, the Schottky barrier disappears, $R_{s}>>R_{j}$, and the spectral density is due to thermal noise related to the series resistance $R_{s}, S_{I}(0) \sim 4 K_{B} T / R_{s}$ [18]. Due to the higher electron mobility of GaAs than GaN semiconductor [19], thermal noise is higher for GaAs than GaN reference SBD. Under applied voltage $V>>V_{b i}, S_{I}(0)$ for GaAs SBD enhances with respect to the prediction of thermal noise, due to the contribution of hot carriers to the low frequency region of the noise spectrum [18]. For GaN SBD hot carriers are not observed because of the high energy gap between the valleys of the conduction band.

\subsection{Returning carriers resonance}

In this subsection we evaluate the dependence of the central frequency and amplitude of the $\mathrm{RC}$ resonance of the noise spectra of GaAs and GaN SBDs with the applied voltage, (figures 5(a) and 5(b)), the doping of the epilayer, (figures 5(c) and 5(d)) and the length of the epilayer, (figures $5(\mathrm{e})$ and $5(\mathrm{f})$ ). Lines in figure 5 correspond to the predictions of the analytical model of [13] and the symbols correspond to the results obtained from MC simulations. In addition, each sub-figure shows the tendency of $f_{R C}$ or $S_{I}\left(f_{R C}\right)$ as a function of different parameters. These expression have been obtained from the analytical model of [13] for homogeneous SBDs. In figures 5(c)-5(f), the applied voltages have been selected as $0.80 \mathrm{~V}$ for GaAs SBD and $0.97 \mathrm{~V}$ for GaN SBD, in such a way that the current flowing through both SBDs is the same.

From the particularization of (1) made in appendix A for homogeneous SBDs, the amplitude of the $\mathrm{RC}$ resonance is proportional to average mobility of the electrons in the diode. The lower electron mobility of GaN [19] is the fundamental reason of the lower amplitude of the RC resonance, $S_{I}\left(f_{R C}\right)$, for GaN SBDs. Besides, the central frequency of the RC resonance, $f_{R C}$, is proportional to $\left(\varepsilon_{r} m^{* 2}\right)^{-1 / 4}$. For voltages applied to GaAs and GaN SBDs such that the Schottky barrier height seen by the electrons in the neutral region of the epilayer, $V_{b i}-V$, is the same in both devices, the ratio between $f_{R C}$ of GaN and GaAs SBDs is $\sim 0.58$, as appendix A shows.

Figure 5 shows that the most effective way to shift the RC resonances to higher frequencies is achieved by higher doping concentrations of the epilayer and by the 

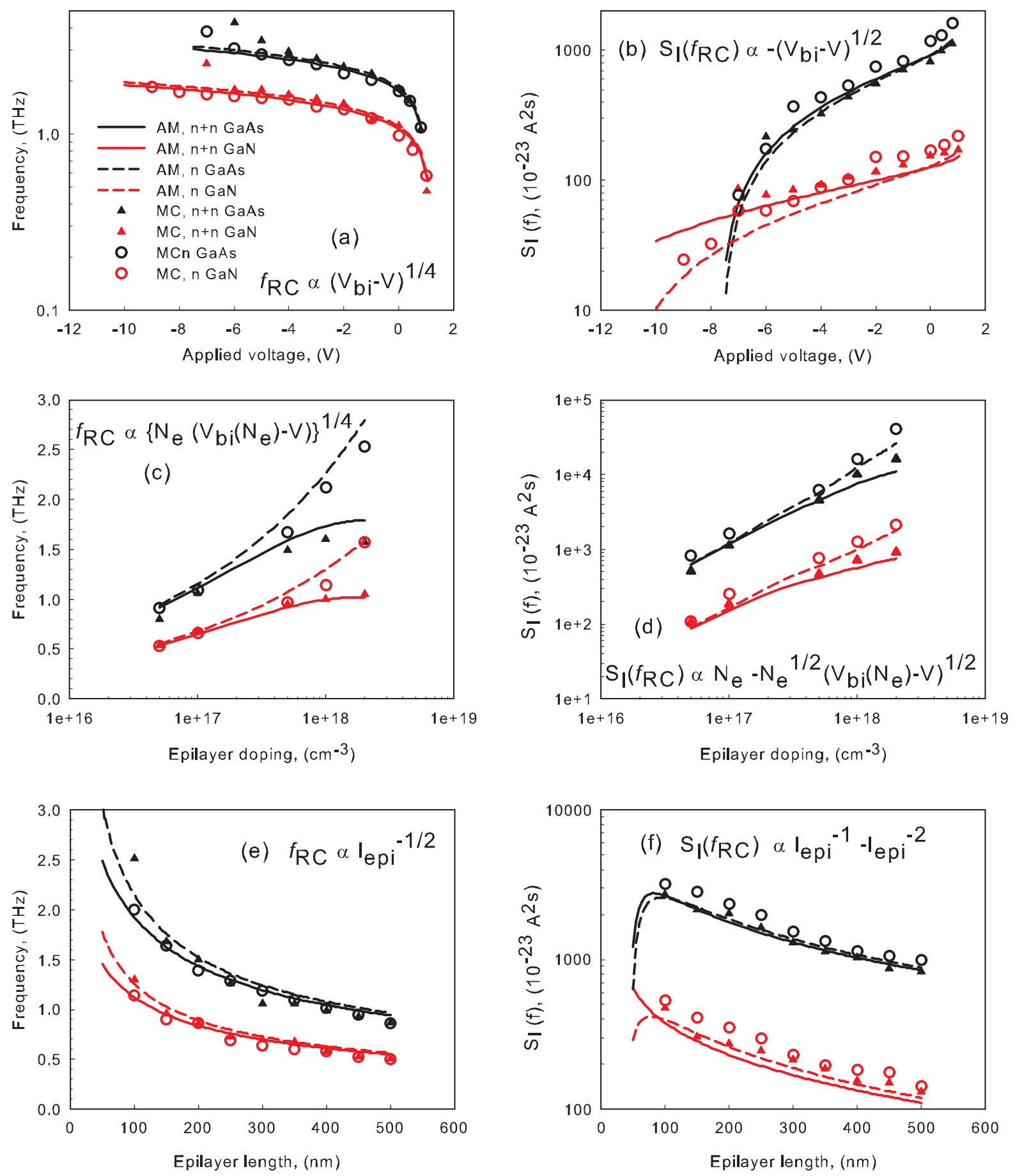

Figure 5. Evolution of the frequency and the amplitude of the RC resonance with the applied voltage ((a), (b)), the doping of the epilayer ((c), (d)) and the length of the epilayer ((e), (f)), for GaAs and GaN. Both $n^{+}-n$ and homogeneous structures are analyzed. Results from MC simulations and the analytical model of [13] $A M$ are shown. In figures 5(c)-5(f), the applied voltages are $0.80 \mathrm{~V}$ for GaAs SBD and $0.97 \mathrm{~V}$ for GaN SBDs in order to obtain the same current. 


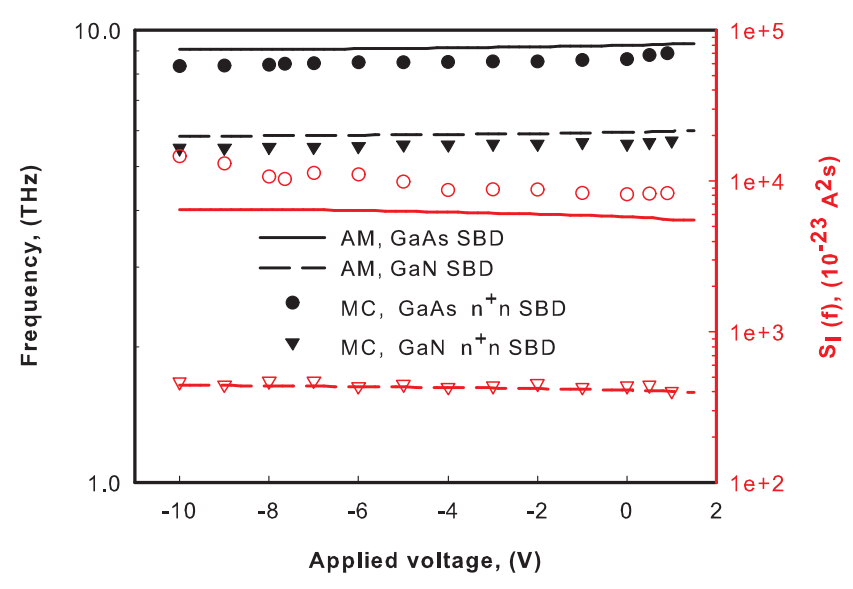

Figure 6. Evolution of the frequency (black data) and the amplitude (red data) of the HP resonance with the applied voltage for GaAs and GaN reference SBDs, from MC simulations (symbols) and the analytical model of [13] AM (lines).

reduction of its length. Besides, from the analysis of the homogeneous SBDs, we observe that for $n^{+}-n$ SBDs the substrate reduces the central frequency of the RC resonance with respect to homogeneous SBDs, especially for SBDs with short and high doped epilayer.

\subsection{Hybrid plasma resonance}

This subsection evaluates the dependence of the HP resonance with the applied voltage, doping and length of the epilayer, employing the analytical model of [13] (continuous lines in figures 7, 8 and 9) together with MC simulations (symbols).

According to (2) the central frequency of the HP resonance is located between the plasma frequencies of the substrate and the epilayer. Since the effective mass of the electrons in GaN is higher than in GaAs, the plasma frequency of the substrate and the epilyer is lower for GaN than GaAs and the central frequency of the HP resonance in GaN is lower than in GaAs based SBDs. The description of the self-consistent coupling of the fluctuations of the carrier velocity and the electric field originated by the $n^{+}-n$ inhomogeneity through the Langevin equation, let the analytical model [13] to predict correctly the performance of the HP resonance. When the doping of the substrate is equal to the epilayer doping, $N_{D, s}=N_{D, e}=10^{17} \mathrm{~cm}^{-3}$, the epilayersubstrate homojunction does not exist and the HP resonance disappears.

Figure 6 shows a low sensitivity of the HP resonance with the applied voltage. Figure 7 and figure 8 present the dependence of the HP peak with the doping and the length of the epilayer, respectively. The results presented in these figures have been obtained under the the same applied voltage of figures $5(\mathrm{c})-5(\mathrm{f})$, corresponding to similar values of current flowing through GaAs and GaN reference SBDs. 


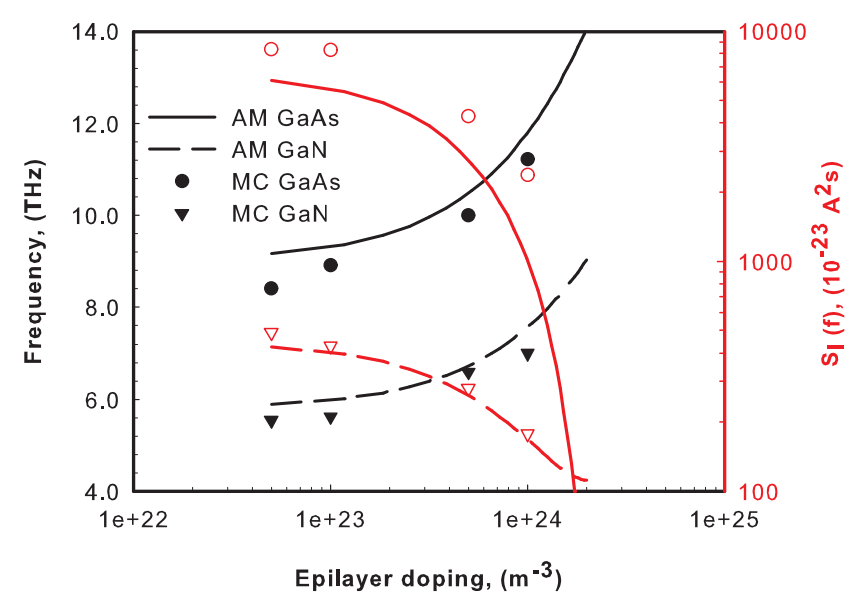

Figure 7. Evolution of the frequency (black data) and the amplitude (red data) of the HP resonance with the doping concentration of the epilayer for GaAs and GaN reference SBDs from MC simulations (symbols) and the analytical model of [13] AM (lines). The applied voltages have been selected as $0.80 \mathrm{~V}$ for GaAs SBD and $0.97 \mathrm{~V}$ for GaN SBD.

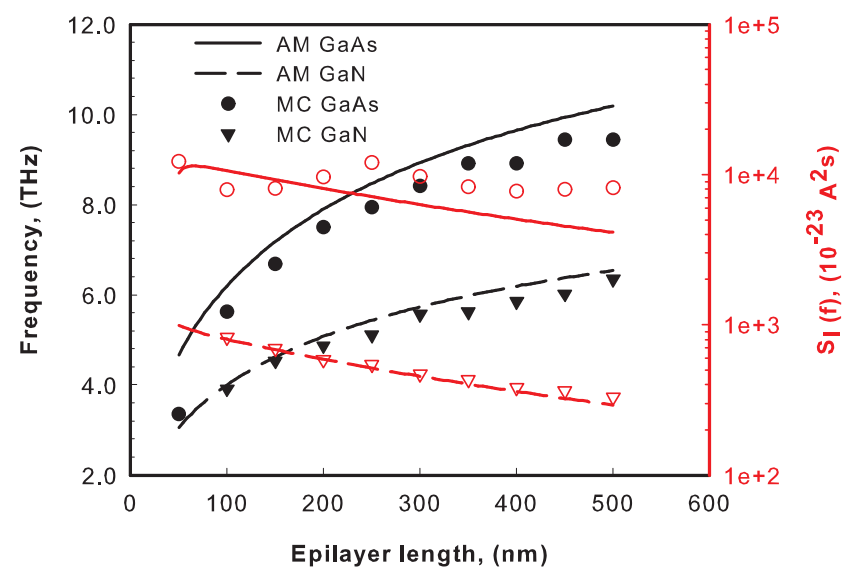

Figure 8. Evolution of the frequency (black data) and the amplitude (red data) of the HP resonance with the length of the epilayer for GaAs and GaN reference SBDs, from MC simulations (symbols) and the analytical model of [13] AM (lines). The applied voltages have been selected as $0.80 \mathrm{~V}$ for GaAs SBD and $0.97 \mathrm{~V}$ for GaN SBD.

\section{Noise under time varying conditions}

In this section we analyze the main features of the noise spectra of SBDs operating under time varying conditions. Subsection 5.1 evaluates the expected $2 q \bar{I}$ performance of the low frequency region of the noise spectrum, where $\bar{I}$ represents the average current flowing in the SBDs. The performance of the RC and the HP resonances is described in subsection 5.2 .

We evaluate the response of GaAs and GaN based SBDs employing MC simulations for two different sinusoidal excitations at different frequencies $f_{e x},\left(V(t)=V_{0}+\right.$ $\left.V_{1} \sin \left(2 \pi f_{e x} t\right)\right)$ : varistor mode defined by a bias $V_{0}=0.8 \mathrm{~V}$ and amplitude $V_{1}=0.1 \mathrm{~V}$, 


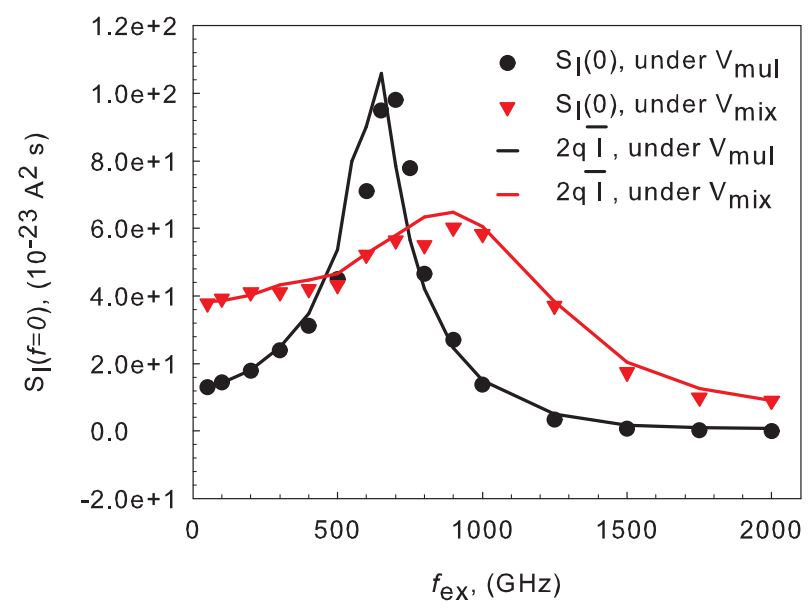

Figure 9. Noise spectral density at $f=0 \mathrm{~Hz}$ and the $2 q \bar{I}$ law for GaAs reference SBD under $V_{m i x}$ and $V_{m u l}$ for different $f_{e x}$.

and varactor mode defined by $V_{0}=0.0 \mathrm{~V}, V_{1}=0.9 \mathrm{~V}$. The former excitation that we denote by $V_{\text {mix }}\left(t, f_{e x}\right)$ is typical of SBD mixers while the latter described signal that we denoted by $V_{\text {mul }}\left(t, f_{e x}\right)$ is typical of SBD multipliers.

\subsection{Low frequency noise}

Figure 9 shows the noise spectral density at $f=0 \mathrm{~Hz}, S_{I}(0)$, obtained from MC simulations for the GaAs reference SBD under applied signals $V_{m i x}\left(t, f_{e x}\right)$ and $V_{\text {mul }}\left(t, f_{\text {ex }}\right)$ for different $f_{\text {ex }}$. Lines in figure 9 , which represent the $2 q \bar{I}$ law for shot noise, show a good agreement with $S_{I}(0)$ calculated with MC. The average current $\bar{I}$, and consequently the $2 q \bar{I}$ noise, increase as the frequency of the applied signal rises, reaching a maximum at frequencies $f_{\text {max }}=650 \mathrm{GHz}$ and $f_{\max }=900 \mathrm{GHz}$ under $V_{\text {mul }}$ and $V_{\text {mix }}$, respectively, and they drop slowly at higher frequencies. While the frequency of the applied signal is lower than $f_{\max }$, the average current flowing through the SBD rises with $f_{e x}$ due to the contribution of the displacement current. When $f_{e x}>f_{\max }$, velocity saturation takes place [20,21], and, therefore, the current through the diode is limited because the depletion region cannot move faster than allowed by the maximum electron velocity $\ddagger$, see appendix B. $f_{\max }$ varies depending on the working conditions, $V_{\text {mix }}\left(t, f_{e x}\right)$ or $V_{\text {mul }}\left(t, f_{e x}\right)$, because of the non-linear dependence of the depletion region length with the applied voltage.

Table 1 shows the average current, $\bar{I}$, and phasor at the fundamental frequency of the current response of the GaAs SBD under $V_{m u l}$ and $V_{m i x}$ for different $f_{\text {ex }}$. The electron velocity saturation results in the reduction of both components as frequency increases.

The velocity saturation effect is more severe for GaN diodes because of its lower mobility. When $f_{e x} \gtrsim 300 \mathrm{GHz}$, the diode performance is limited by the velocity $\ddagger$ The displacement current in the depleted region of the epilayer must be matched by the electron conduction current through the non-depleted semiconductor 
Table 1. Average current and fundamental phasor of the current response for the GaAs reference SBD under $V_{m u l}$ and $V_{m i x}$ for different $f_{e x}$.

\begin{tabular}{|c|c|c|c|c|}
\hline \multirow[b]{2}{*}{$\begin{array}{l}f_{e x}, \\
(\mathrm{GHz})\end{array}$} & \multicolumn{2}{|r|}{$V_{m u l}$} & \multicolumn{2}{|r|}{$V_{m i x}$} \\
\hline & $\begin{array}{l}\bar{I}, \\
(\mathrm{~mA})\end{array}$ & $\begin{array}{l}I_{1}, \\
(\mathrm{~mA})\end{array}$ & $\begin{array}{l}\bar{I} \\
(\mathrm{~mA})\end{array}$ & $\begin{array}{l}I_{1}, \\
(\mathrm{~mA})\end{array}$ \\
\hline 50 & 0.41 & $11.6 \mathrm{e}^{-\mathrm{i} 0.027 \pi}$ & 1.19 & $3.6 \mathrm{e}^{-\mathrm{i} 0.120 \pi}$ \\
\hline 100 & 0.45 & $23.3 \mathrm{e}^{-\mathrm{i} 0.023 \pi}$ & 1.2 .0 & $6.3 \mathrm{e}^{-\mathrm{i} 0.129 \pi}$ \\
\hline 300 & 0.77 & $71.3 \mathrm{e}^{-\mathrm{i} 0.045 \pi}$ & 1.35 & $18.6 \mathrm{e}^{-\mathrm{i} 0.124 \pi}$ \\
\hline 600 & 2.81 & $143.0 \mathrm{e}^{-\mathrm{i} 0.115 \pi}$ & 1.64 & $38.9 \mathrm{e}^{-\mathrm{i} 0.217 \pi}$ \\
\hline 900 & 0.77 & $118.0 \mathrm{e}^{-\mathrm{i} 0.224 \pi}$ & 2.03 & $57.1 \mathrm{e}^{-\mathrm{i} 0.376 \pi}$ \\
\hline 1250 & 0.15 & $123.0 \mathrm{e}^{-\mathrm{i} 0.230 \pi}$ & 1.19 & $54.5 \mathrm{e}^{-\mathrm{i} 0.571 \pi}$ \\
\hline 1500 & 0.05 & $127.1 \mathrm{e}^{-\mathrm{i} 0.226 \pi}$ & 0.64 & $43.3 \mathrm{e}^{-\mathrm{i} 0.656 \pi}$ \\
\hline 1750 & 0.03 & $130.2 \mathrm{e}^{-\mathrm{i} 0.220 \pi}$ & 0.39 & $32.9 \mathrm{e}^{-\mathrm{i} 0.702 \pi}$ \\
\hline 2000 & 0.02 & $133.2 \mathrm{e}^{-\mathrm{i} 0.208 \pi}$ & 0.28 & $24.6 \mathrm{e}^{-\mathrm{i} 0.721 \pi}$ \\
\hline
\end{tabular}

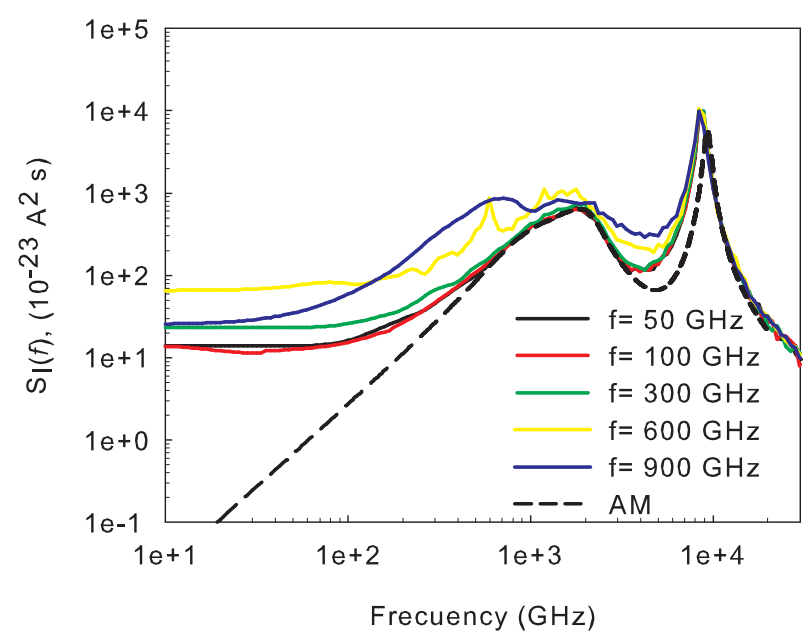

Figure 10. Noise spectral density of GaAs reference SBDs under $V_{m u l}$ for different frequencies.

saturation, appendix B.

\subsection{Medium-high frequency noise}

Figure 10 and figure 11 show the noise spectra obtained from MC simulations of GaAs and GaN reference SBDs respectively, under excitation $V_{m u l}\left(t, f_{e x}\right)$ for different $f_{e x}$. Black dashed lines have been obtained with the analytical model of [13] as a superposition of noise spectra of the SBDs under static conditions with a sinusoidal distribution between $V_{\min }=V_{0}-V_{1}$ and $V_{\max }=V_{0}+V_{1}$.

Figure 10 shows that the noise spectra obtained from MC simulations of GaAs SBD under applied signals $V_{m u l}$ with $f_{e x} \lesssim 300 \mathrm{GHz}$ can be correctly described as a superposition of noise spectra under static conditions (black dashed line). The noise spectra for $f_{e x}=600 \mathrm{GHz}$ and $900 \mathrm{GHz}$ show a resonance at the frequency of the 


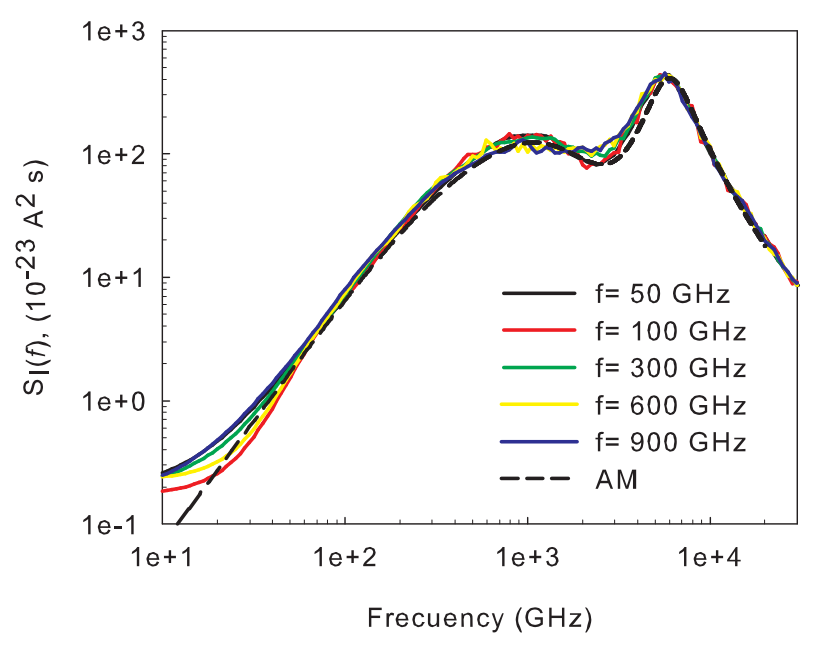

Figure 11. Noise spectral density of GaN reference SBDs under $V_{m u l}$ for different frequencies.

applied signal and an enhancement of the noise level in the frequency region near $f_{e x}$. Under these conditions, the electric field inside the diode is high enough to give rise to intervalley transitions between $\Gamma$ and $L$ valleys. This process generates two groups of carries in the GaAs SBD characterized by different effective mass and different mobilities. According to the analysis performed in [10], when the frequency $f_{e x}$ of the applied signal is higher than the frequency associated to the transitions between the two groups of carriers, $(\sim 500 \mathrm{GHz}[10])$, these transitions give rise to a resonance in the noise spectrum at the frequency of the applied signal and higher levels of noise. The resonance predicted by [10] is clearly observed in figure 10 for $f_{e x}=600 \mathrm{GHz}$. At $900 \mathrm{GHz}$ the ratio between the amplitude and the frequency of the applied signal overpasses the optimum value for the generation of the peak associated to the intervalley transitions and the amplitude of this resonance decreases and its frequency is shifted [10].

The high energy gap of GaN [19] prevents GaN SBD from intervalley transitions under conditions $V_{m u l}$, as figure 11 shows. Consequently the related noise spectra obtained from the MC simulations are correctly described as a superposition of noise spectra under static conditions, figure 11.

From the MC simulation of the GaAs and GaN SBD under excitations $V_{m i x}\left(t, f_{e x}\right)$ for different $f_{e x}$, the small amplitude of the applied signal is insufficient to give rise to intervalley transitions in the semiconductor and the noise spectra obtained from the MC simulations can be described as a superposition of noise spectra obtained under static conditions.

The HP resonance observed in these figures, for GaAs and GaN SBDs, is understood as a superposition of noise spectra under static conditions, i.e. it does not vary with the frequency of the simulated excitations.

The analysis performed in this section has shown that the noise spectra in GaAs and GaN SBDs operating under time varying conditions can be described as a superposition of noise spectra under static conditions while hot carrier effects are not present. 


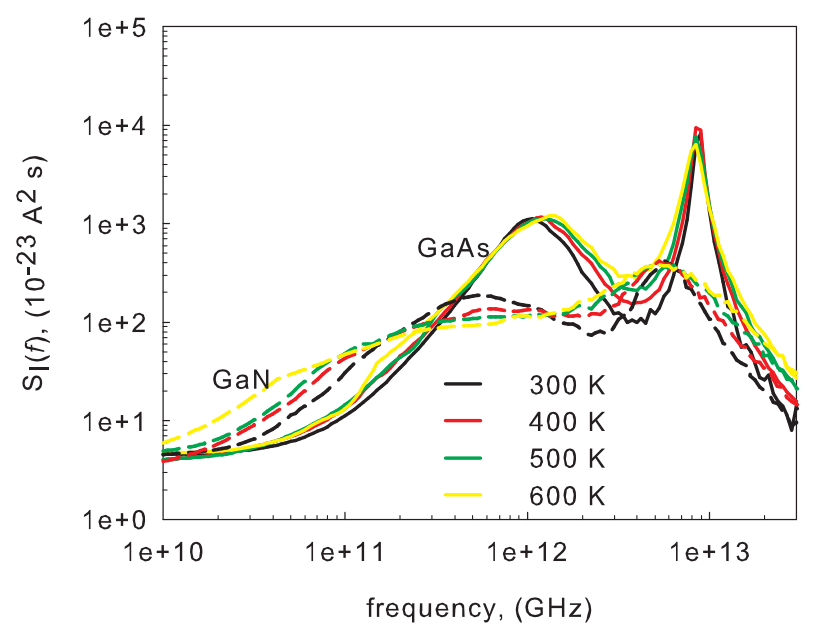

Figure 12. Noise spectra obtained from MC simulations of the GaAs and GaN SBDs under constant voltage conditions and temperatures from $300 \mathrm{~K}$ to $600 \mathrm{~K}$. The applied voltages have been selected to obtain similar values of current $(\sim 0.13 \mathrm{~mA})$ in GaAs and GaN SBDs at all the simulated temperatures.

Consequently, the steps given in subsection 4.2 to reduce the noise level of SBDs under static conditions in the near $\mathrm{THz}$ region, are valid under time varying operation of the devices.

\section{Temperature dependence of the noise spectra}

To analyze the impact of self-heating on noise spectra, this section evaluates the temperature dependence of the low frequency and the medium-high frequency noise for GaAs and GaN SBDs as a function of room temperature.

\subsection{Noise spectra under static conditions}

Figure 12 shows the temperature dependence of the noise spectra obtained from MC simulations of the GaAs and GaN SBDs under static conditions. The applied voltages have been selected to obtain similar values of current in GaAs and GaN SBDs at all the simulated temperatures $T(\sim 0.13 \mathrm{~mA}$, figure 3$)$. These applied voltages are lower than $V_{b i}(T)$ and, therefore, the low frequency noise is described by the $2 q I$ law for shot noise.

Under these conditions, the width of the depletion region for GaAs and GaN SBDs increases with $T$ and according to (3) and (2), the central frequency of the RC resonance increases and the central frequency of the HP resonance decreases with increasing temperature $\left(f_{R C} \sim r_{d}\right.$ and $f_{H P} \sim \gamma-r_{d}$ where $\gamma$ represents a factor independent of $T$, figure 2 and (2)). The variations of the noise spectra with the temperature are more pronounced for GaN than GaAs SBDs because of the different performance of the electron mobility with the temperature [19]. 


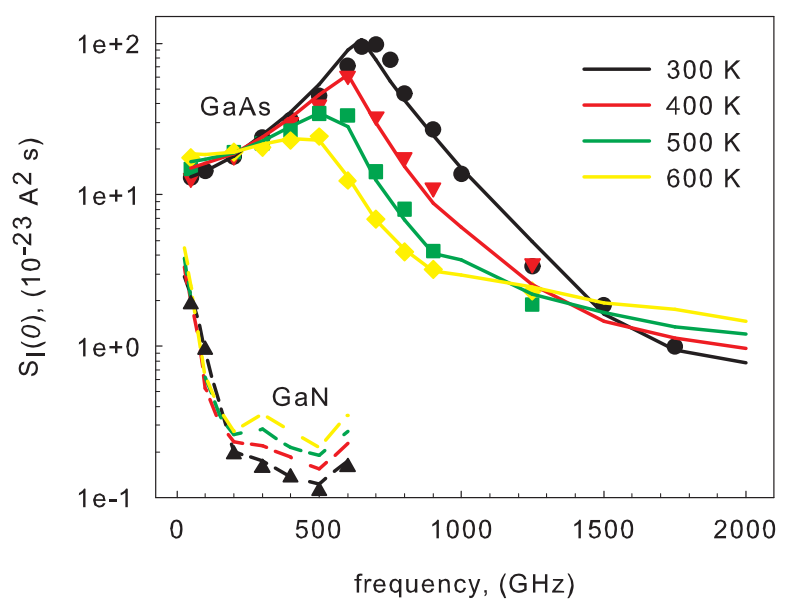

Figure 13. Noise spectral density at $f=0 \mathrm{~Hz}$ and the $2 q \bar{I}$ law for GaAs and GaN SBDs under applied signals of amplitude corresponding to the maximum DC voltage without entering the ohmic regime at each simulated temperature, bias point $0 \mathrm{~V}$ and different frequencies.

\subsection{Noise under time varying conditions}

The results of the study for the temperature dependence of the low frequency noise of the SBDs under time varying conditions are shown in figure 13. This figure shows the noise spectral density at $f=0 \mathrm{~Hz}, S_{I}(0)$, for the GaAs and GaN SBDs as a function of the frequency of the applied signal and temperatures from $300 \mathrm{~K}$ to $600 \mathrm{~K}$. The bias point is $0 \mathrm{~V}$ and the amplitude of the RF signal, $V_{1}$, corresponds to the maximum DC voltage for the GaAs and GaN SBDs at each temperature without entering the ohmic regime, figure 3 . The results of $S_{I}(0)$ from $\mathrm{MC}$ simulations show a good agreement with the $2 q \bar{I}$ law for shot noise (continuous and dashed lines of figure 13).

For GaAs SBD, the peak of $S_{I}(0)$, which is related to current velocity saturation and is described in subsection 5.1, shifts to lower frequencies as the temperature increases because of the reduction of the electron mobility with temperature [19]. The reduction of the amplitude of this peak with temperature is due to the increment of the series resistance of the SBD and the reduction of the nonlinear capacitance with the increment of $T$, figure 3. GaN SBD performance is dominated by a higher series resistance even at low frequencies.

Regarding the medium-high frequency range, the noise performance can be reproduced by the analytical model of [13] as a superposition of noise spectra under static conditions up to $\mathrm{THz}$ regime for GaN SBDs, and up to $300 \mathrm{GHz}$ for GaAs SBDs, section 5.2. Figure 14 shows the temperature dependence of the medium-high frequency noise of the GaAs and GaN SBDs under applied signals of frequency $600 \mathrm{GHz}$. At this frequency and higher, the intervalley transitions between $\Gamma$ and $L$ valleys in the GaAs SBD described in subsection 5.2 appear, giving rise to a peak at the frequency of the applied signal. As $T$ increases, the average energy of the carriers increases, and the average occupation of the L-valley increases. Therefore, the amplitude of the peak 


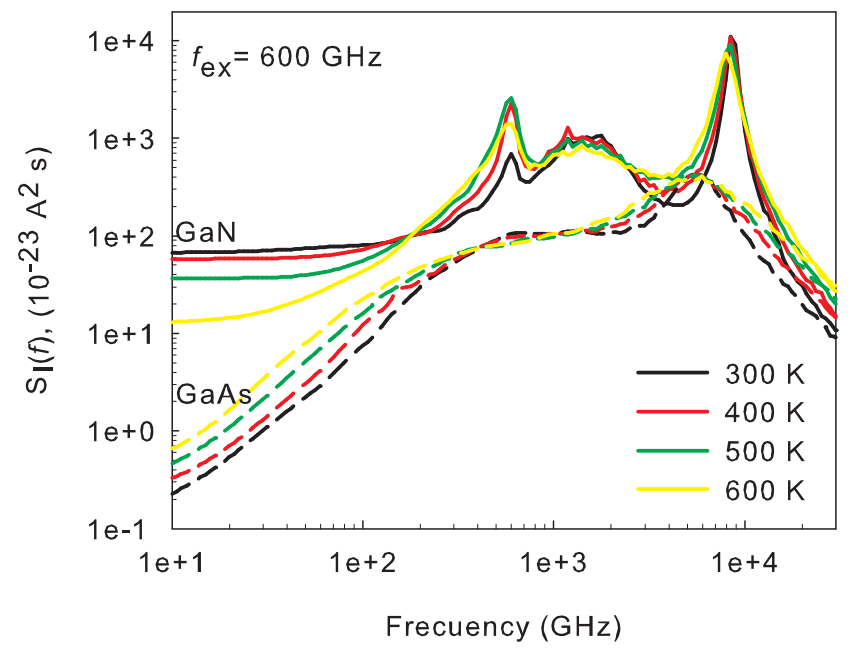

Figure 14. Noise spectral density of the GaAs and GaN SBDs under applied signals simulated to obtain the results of figure 13 at $600 \mathrm{GHz}$.

associated to these transitions rises according to [10].

\section{Conclusions}

This paper has presented a systematic study of the noise features of GaAs and GaN SBDs operating under static and time varying conditions based on Monte Carlo method. Low frequency mechanisms like shot and thermal noise and high frequency mechanisms like returning carriers and plasma phenomena have been characterized as a function of device structure, temperature, and working regimes. Contributions of hot electrons to noise have been also discussed.

Besides, this paper has validated analytical models available in the literature to describe the noise spectra of SBDs under very different working regimes, with emphasis on the limits of application of these models. This is an initial step towards the development of numerical physical device models based on partial differential equations which will integrate noise together with electrical performance. These models will be also integrated into circuital simulators for a joint optimization of the device structure and the embedding circuit.

\section{Acknowledgments}

This work was supported by the Junta de Castilla y Leon, Spanish National Research and Development Program under projects GR270, SA019A08, TEC2008-02148, TEC201015413, TeraSense (Consolider- Ingenio 2010, CDS2008-00068), RooTHz (Eurpean Comission- ICT-2009-243845) 
Table A1. Parameter of GaAs and GaN semiconductor employed in this work.

\begin{tabular}{lll}
\hline & GaAs & GaN \\
\hline$\varepsilon_{r}$ & 12.9 & 8.9 \\
$m^{*}$ & 0.063 & 0.222 \\
$\mu_{e},\left(m^{2} /(\mathrm{Vs})\right)$ & $0.5068^{\mathrm{a}}$ & $0.0674^{\mathrm{a}}$ \\
$\mu_{s},\left(m^{2} /(\mathrm{Vs})\right)$ & $0.2701^{\mathrm{b}}$ & $0.0338^{\mathrm{b}}$ \\
\hline
\end{tabular}

${ }^{a}$ From MC simulation of bulk semiconductor doped $10^{17} \mathrm{~cm}^{-3}$ at $300 \mathrm{~K}$.

b From MC simulation of bulk semiconductor doped $210^{18} \mathrm{~cm}^{-3}$ at $300 \mathrm{~K}$.

\section{Appendix A. Equations comparing the RC resonance for GaAs and GaN SBDs}

The following equations present the ratios of the central frequency, $f_{R C}$, the amplitude, $S_{I}\left(f_{R C}\right)$, and the bandwidth at $-3 \mathrm{~dB}, \Delta f_{R C}$, for the $\mathrm{RC}$ resonance of GaN and GaAs SBDs. They have been obtained from equations (2) and (3).

$$
\begin{aligned}
& \rho_{f}=f_{R C, G a N} / f_{R C, G a A s}=\left[\frac{\left(m^{* 2} \varepsilon_{r}\right)_{G a A s}}{\left(m^{* 2} \varepsilon_{r}\right)_{G a N}} \frac{\left(V_{b i}-V\right)_{G a N}}{\left(V_{b i}-V\right)_{G a A s}}\right]^{1 / 4} \\
& \simeq 0.58\left[\frac{\left(V_{b i}-V\right)_{G a N}}{\left(V_{b i}-V\right)_{G a A s}}\right]^{1 / 4} \\
& \rho_{S}=S_{I}\left(f_{R C, G a N}\right) / S_{I}\left(f_{R C, G a A s}\right)=\frac{\left(\varepsilon_{r} m^{*} \mu\right)_{G a N}}{\left(\varepsilon_{r} m^{*} \mu\right)_{G a A s}^{2}} \frac{1-\left(f_{p e} / f_{R C}\right)_{G a N}^{2}}{1-\left(f_{p e} / f_{R C}\right)_{G a A s}^{2}} \\
& \simeq 2.43 \frac{\left.\mu\right|_{G a N}}{\left.\mu\right|_{G a A s}} \rho_{f}^{2} \frac{1-\left(f_{p e} / f_{R C}\right)_{G a N}^{2}}{1-\left(f_{p e} / f_{R C}\right)_{G a A s}^{2}} \\
& \rho_{\Delta}=\Delta f_{R C, G a N} / \Delta f_{R C, G a A s}=\frac{1}{\rho_{f}} \frac{\left[\sqrt{f_{s c}^{4}+\left(4 \pi f_{s c} f_{R C}\right)^{2}}\right]_{G a N}}{\left[\sqrt{f_{s c}^{4}+\left(4 \pi f_{s c} f_{R C}\right)^{2}}\right]_{G a A s}}
\end{aligned}
$$

where $\Delta f_{R C}=f_{b}-f_{a}=\left(f_{b}^{2}-f_{a}^{2}\right) /\left(f_{a}+f_{b}\right) \simeq\left(\left(f_{s c}^{4}+\left(4 \pi f_{s c} f_{R C}\right)^{2}\right)^{1 / 2}\right) /\left(2 f_{R C}\right) ; f_{a}$ and $f_{b}$ are frequencies such that $S_{I}\left(f_{a}\right)=S_{I}\left(f_{b}\right)=S_{I}\left(f_{R C}\right) / 2$ and $f_{a}<f_{R C}<f_{b}$. The ratios $\rho_{S}$ and $\rho_{\Delta}$ are valid only for homogeneous SBDs. The particularization of (1) for homogeneous SBDs and its evaluation at $f_{R C}, S_{I}\left(f_{R C}\right)=4 k_{B} T 2 \pi C\left(f_{p e}^{2}-f_{R C}^{2}\right) / f_{s c}=$ $4 k_{B} T q N_{e} A \mu\left(1-r_{d}\right)$, where $\mu$ is the low field electron mobility, have been employed to obtain these ratios.

Table A1 shows the material parameters we have used when dealing with the analytical model of [13].

$\mu_{e}$ and $\mu_{s}$ of table A1 represent the low field electron mobilities obtained from MC simulation of bulk GaAs and GaN semiconductors at $300 \mathrm{~K}$ with doping concentrations equal to the doping concentrations of the epilayer and the substrate of the reference SBD respectively. The rest of parameters presented in this table can be found in $[14,22]$. 


\section{Appendix B. Velocity saturation}

In Schottky diodes the displacement current through the depletion region, related to the nonlinear capacitance of the Schottky junction $i_{d}=q N_{e} A \mathrm{~d} W(t) / \mathrm{d} t$, must match the electron conduction current $i_{c}=q N_{e} A v_{e}$ in the non-depleted semiconductor. In the expressions for $i_{d}$ and $i_{c}, W(t)$ represents the length of the depletion region, $\mathrm{A}$ is the cross-section area of the diode, $N_{e}$ is the doping concentration of the epilayer, $V_{b i}$ is the built-in potential and $v_{e}$ is the electron velocity. According to [20], the edge of the depletion region cannot move faster than allowed by the maximum electron velocity in the non-depleted semiconductor, i.e.

$$
\frac{\mathrm{d} W(t)}{\mathrm{d} t}<v_{e, \max }
$$

where $v_{e, \max }$ is the maximum electron velocity, $v_{e, \max }=\mu(E) E, \mu$ is the electron mobility as a function of the electric field $E$ in the non-depleted semiconductor. Since the speed of the electrons in the semiconductor cannot exceed certain limits, $v_{e, \text { max }}$, a current limiting phenomenon or saturation phenomenon is expected with the increasing frequency or amplitude of the signal applied to the diode.

Besides, the finite acceleration of the electrons in the diodes introduces a time delay between the applied signal and the response of the electrons. This inertial effect can be modeled by an inductive performance of the SBD [20]

$$
2 \pi f_{e x} H_{e p i}=R_{e p i} f_{e x} / f_{s c}
$$

where $H_{e p i}$ represents the inductance associated to inertia of the electrons, $f_{e x}$ is the frequency of the applied signal, $R_{e p i}$ is the series resistance of the non-depleted epilayer and $f_{s c}$ is the carrier momentum relaxation rate. $f_{s c}$ is of the order of $0.88-1.11 \mathrm{THz}$ for GaAs semiconductor, $\left(N_{e}=10^{17} \mathrm{~cm}^{-3}, \mu \sim 0.5-0.4 \mathrm{~m}^{2} / V s\right)$ and $1.85-2.14 \mathrm{THz}$ for GaN semiconductor, $\left(N_{e}=10^{17} \mathrm{~cm}^{-3}, \mu \sim 0.068-0.059 \mathrm{~m}^{2} / \mathrm{Vs}\right)$ at $300 \mathrm{~K}$.

To evaluate the impact of these phenomena in the performance of the GaAs and GaN reference SBDs under time varying regimes considered in this paper, table B1 shows the phasor at the fundamental frequency of $W(t)$, obtained from MC simulations. The last row of this table includes the phasor of the width of the depletion region calculated through the theoretical expression $l_{d}(t)=\left(2 \varepsilon_{0} \varepsilon_{r}\left(V_{b i}-V(t) / q / N_{e}\right)\right)^{1 / 2}$. Figure B1 represents the evolution of $W(t)$ in a period of the applied signal for the GaAs SBD under conditions $V_{\text {mul }}$ for different frequencies.

For GaAs SBD under conditions $V_{m u l}$ and $V_{m i x}$ table B1 shows the reduction of the amplitude of the phasors of $\mathrm{W}(\mathrm{t})$ with respect to the amplitude of the phasor of $l_{d}(t)$, (last row of table B1), for frequencies of the applied signal higher than $f_{\text {max }}=600 \mathrm{GHz}$ and $f_{\max }=900 \mathrm{GHz}$, respectively. This performance is due to the velocity saturation of the electrons in the non-depleted semiconductor. Due to the non-linear dependence of the depletion region length with the applied voltage, $f_{\max }$ depends on the working conditions.

Because of the low electron mobility of GaN, the electron velocity saturation in 
Table B1. Phasor at the fundamental frequency of the evolution of the width of the depletion region in a period of the applied signal for GaAs and GaN reference SBDs under $V_{m u l}$ and $V_{m i x}$ for different frequencies.

\begin{tabular}{|c|c|c|c|c|}
\hline \multirow[b]{2}{*}{$f,(\mathrm{GHz})$} & \multicolumn{2}{|c|}{ GaAs } & \multicolumn{2}{|c|}{$\mathrm{GaN}$} \\
\hline & $W_{V_{m u l}},(\mathrm{~nm})$ & $W_{V_{m i x}},(\mathrm{~nm})$ & $W_{V_{m u l}},(n m)$ & $W_{V_{m i x}},(\mathrm{~nm})$ \\
\hline 50 & $62.62 \mathrm{e}^{\mathrm{i} 0.529 \pi}$ & $16.72 \mathrm{e}^{\mathrm{i} 0.524 \pi}$ & $44.86 \mathrm{e}^{\mathrm{i} 0.514 \pi}$ & $9.00 \mathrm{e}^{\mathrm{i} 0.476 \pi}$ \\
\hline 100 & $62.05 \mathrm{e}^{\mathrm{i} 0.521 \pi}$ & $16.28 \mathrm{e}^{\mathrm{i} 0.499 \pi}$ & $44.56 \mathrm{e}^{\mathrm{i} 0.477 \pi}$ & $8.67 \mathrm{e}^{\mathrm{i} 0.435 \pi}$ \\
\hline 300 & $64.20 \mathrm{e}^{\mathrm{i} 0.492 \pi}$ & $16.89 \mathrm{e}^{\mathrm{i} 0.438 \pi}$ & $40.25 \mathrm{e}^{\mathrm{i} 0.349 \pi}$ & $7.29 \mathrm{e}^{\mathrm{i} 0.271 \pi}$ \\
\hline 600 & $68.65 \mathrm{e}^{\mathrm{i} 0.415 \pi}$ & $19.02 \mathrm{e}^{\mathrm{i} 0.327 \pi}$ & $29.78 \mathrm{e}^{\mathrm{i} 0.168 \pi}$ & $4.84 \mathrm{e}^{\mathrm{i} 0.073 \pi}$ \\
\hline 900 & $29.54 \mathrm{e}^{\mathrm{i} 0.057 \pi}$ & $20.46 \mathrm{e}^{\mathrm{i} 0.136 \pi}$ & $21.36 \mathrm{e}^{\mathrm{i} 0.050 \pi}$ & $3.36 \mathrm{e}^{-\mathrm{i} 0.025 \pi}$ \\
\hline 1250 & $20.39 \mathrm{e}^{-\mathrm{i} 0.064 \pi}$ & $15.22 \mathrm{e}^{-\mathrm{i} 0.073 \pi}$ & $15.67 \mathrm{e}^{-\mathrm{i} 0.061 \pi}$ & $2.33 \mathrm{e}^{-\mathrm{i} 0.091 \pi}$ \\
\hline 1500 & $18.21 \mathrm{e}^{-\mathrm{i} 0.160 \pi}$ & $10.21 \mathrm{e}^{-\mathrm{i} 0.158 \pi}$ & $12.95 \mathrm{e}^{-\mathrm{i} 0.151 \pi}$ & $2.19 \mathrm{e}^{-\mathrm{i} 0.155 \pi}$ \\
\hline 1750 & $14.76 \mathrm{e}^{-\mathrm{i} 0.209 \pi}$ & $7.73 \mathrm{e}^{-\mathrm{i} 0.226 \pi}$ & $10.86 \mathrm{e}^{-\mathrm{i} 0.200 \pi}$ & $1.15 \mathrm{e}^{-\mathrm{i} 0.219 \pi}$ \\
\hline 2000 & $13.09 \mathrm{e}^{-\mathrm{i} 0.305 \pi}$ & $5.92 \mathrm{e}^{-\mathrm{i} 0.208 \pi}$ & $8.92 \mathrm{e}^{-\mathrm{i} 0.249 \pi}$ & $1.15 \mathrm{e}^{-\mathrm{i} 0.469 \pi}$ \\
\hline$\overline{l_{d}}$ & $62.92 \mathrm{e}^{\mathrm{i} 0.531 \pi}$ & $15.98 \mathrm{e}^{\mathrm{i} 0.531 \pi}$ & $45.88 \mathrm{e}^{\mathrm{i} 0.531 \pi}$ & $8.95 \mathrm{e}^{\mathrm{i} 0.531 \pi}$ \\
\hline
\end{tabular}

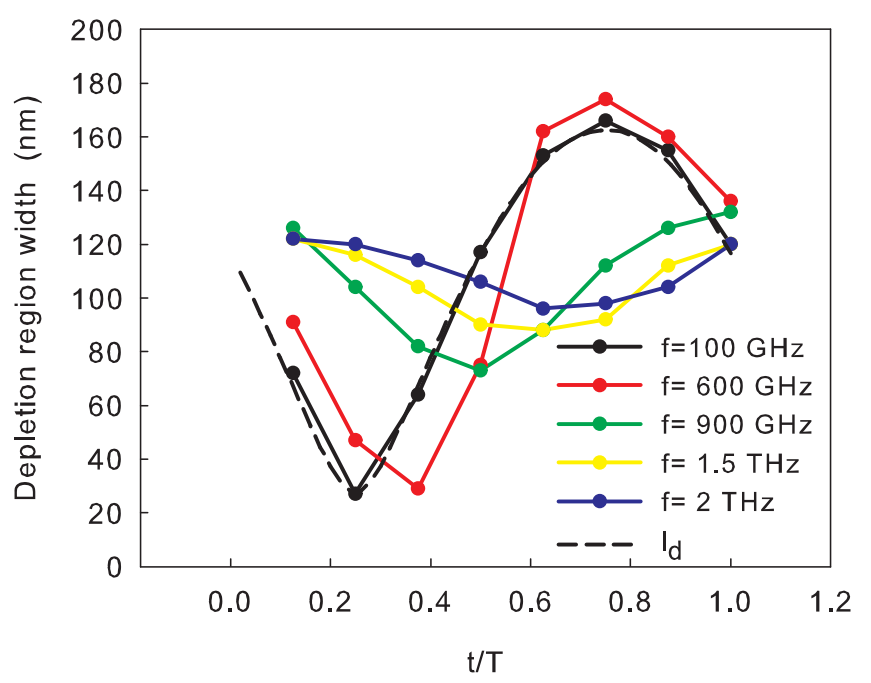

Figure B1. Evolution of the width of the depletion region of the GaAs reference SBD in a period of the applied signal $V_{m u l}$, for different frequencies.

GaN SBD appears at frequencies higher than $300 \mathrm{GHz}$ under both simulated conditions $V_{m u l}$ and $V_{m i x}$, as table B1 shows.

The phase of the phasors of $W(t)$ shown in table B1 for both GaAs and GaN SBDs delays with respect to the phase of the phasor of $l_{d}(t)$, (last row of table B1), with the increment of the frequency of the applied signal. This phase delay is the consequence of the inertia of the electrons in the non-depleted semiconductor.

\section{References}

[1] Siegel P H 2004 IEEE Trans. Microwave Theory Tech. 52 2438-47

[2] Siegel P H 2007 IEEE Trans. Antennas Propag 55 2957-65 
[3] Dengler R J, Cooper K B, Chattopadhyay G, Mehdi I, Schlecht E, Skalare A, Chen C and Siegel P H 2007 IEEE/MTT-S Int. Microwave Symp. Dig. p 1371

[4] Crowe T W, Bishop W L, Porterfield D W, Hesler J L and Weikle II R M 2005 IEEE Journal of Solid-State Circuits 40 2104-10

[5] Maestrini A, Ward J S, Gill J J, Lee C, Thomas B, Lin R H, Chattopadhyay G and Mehdi I 2010 IEEE Trans. Microwave Theory Tech. 58 1925-32

[6] Thomas B, Maestrini A, Gill J, Lee C, Lin R, Mehdi I, and de Maagt P 2010 IEEE Trans. Microwave Theory Tech. 58 1917-24

[7] Maestrini A, Thomas B, Wang H, Jung C, Treuttel J, Jin Y, Chattopadhyay G, Mehdi I and Beaudin G 2010 C. R. Physique 11 480-95

[8] Siles J V and Grajal J 2008 Proc. of the 19th Int. Symp. on Space Terahertz Technology (Groningen) p 504

[9] der Ziel V 1970 Noise; sources, characterization, measurement (New Jersey: Prentice-Hall)

[10] Shiktorov P, Starikov E, Gružnskis V, Pérez S, González T, Reggiani L, Varani L and Vaissière J C 2003 Phys. Rev. B 67165201

[11] Pérez S, González T, Shiktorov P, Starikov E, Gružinskis V, Reggiani L, Varani L and Vaissière J C 2004 Proc. of the SPIE vol 5470 p 322

[12] Shiktorov P, Starikov E, Gružinskis V, Pérez S, González T, Reggiani L, Varani L and Vaissière J C 2004 IEEE Electron Devices Letters 25 1-3

[13] Shiktorov P, Starikov E, Gružinskis V, Reggiani L, Varani L and Vaissière J L 2005 IEEE Electron Devices Letters 26 2-4

[14] Fischetti M V and Laux S E 1988 Phys. Rev. B 38 9721-45

[15] Mansour N S, Diff K and Brennan K F 1991 J. Appl. Phys. 70 6854-9

[16] Trippe M, Bosman G and Van Der Ziel A 1986 IEEE Trans. Microwave Theory Tech. 34 $1183-92$

[17] Siles J V and Grajal J 2010 IEEE Trans. Microwave Theory Tech. 58 1933-42

[18] González T, Pardo D, Reggiani L and Varani L 1997 J. Appl. Phys. 82 2349-58

[19] Schwierz F 2005 Solid-State Electronics 48 889-95

[20] Kollberg E L, Tolmunen T J, Frerking M A and East J R 1992 IEEE Trans. Microwave Theory Tech. 40 831-8

[21] Grajal J, Krozer V, González E, Maldonado F and Gismero J 2000 IEEE Trans. Microwave Theory Tech. 48 700-11

[22] Madelung O 2004 Semiconductor: data handbook (Berlin: Springer) 